\title{
¿Representa el programa de maquiladoras mexicanas una verdadera estrategia de desarrollo?
}

\author{
Leslie Sklair \\ Centro de Estudios Mexicoamericanos \\ Universidad de California-San Diego
}

\section{Resumen}

El cuestionamiento con que se presenta este artículo propone un esquema evaluativo para la estrategia de la industrialización vía maquiladoras en el caso de México. Parte de señalar que la estrategia de crecimiento basada en la inversión y tecnología extranjera ha adquirido una popularidad inusitada entre los tomadores de decisiones en países del Tercer Mundo. El concepto de dependencia ha sido sometido ya a rigurosas críticas teóricas, sin embargo, lo realmente importante es "establecer el potencial del desarrollo dependiente para ser revertido". El panorama que se ofrece es diverso y abarca aspectos que resultan polémicos e inagotables, y las conclusiones a las que se puede llegar después de reflexionar en el tema, con seguridad no tendrán la misma validez hoy que mañana.

Palabras clave: estrategia de desarrollo, maquiladoras, inversión extranjera, países del tercer mundo, dependencia económica.

\begin{abstract}
The questions presented in this article propose an appraisal scheme for the strategy of industrialization through maquila industry in Mexico. First, it points out that the strategy of growth, based on investment and foreign technology, has become unexpectedly popular among those leaders who take the decisions at countries of the Third World. The concept of dependence has been already submitted to hard theoretical critics; however, what's really important is "to establish the potential of dependent development to push it back". It shows a picture that is varied and has controversial and endless aspects, and after having deepened on the topic, the conclusions will not have the same validity today than in the future.
\end{abstract}

Keywords: development strategy, maquila industry, foreign investment, countries of the third world, economical dependence. 


\title{
¿REPRESENTA EL PROGRAMA DE LAS MAQUILADORAS MEXICANAS UNA VERDADERA ESTRATEGIA DE DESARROLLO?*
}

\author{
Por: \\ Leslic Sklair"*
}

En todos los países más pobres del Tercer Mundo, la creencia en que la industrialización exportadora acelerada por la inversión cxtranjera y la tecnologia (cn adelante IEAIET), ha ganado una resonante victoria sobre las otras estrategias disponibles, tales como la sustitución de importaciones, variados grados de autarquía o la confianzatradicional en la exportación de productos primarios. Aquíno puedo profundizar en las causas que expliquen la hegemonía global de la IEAIET; sin embargo, se intentará evaluar sus consecuencias para México y en particular para la industria maquiladora.

La relación entre desarrollo e inversión extranjera ha sido caracterizada de diversas maneras, pero la que es más útil para mis propósitos es el debate alrededor del controversial asunto de la dependencia. Sabemos de la gran cantidad de excelentes críticasacerca de la noción de dependencia y cómo se ha usado y abusado de ella en la literatura del desarrollo económico. Aquí quisiera usar el término en un sentido específico y desapasionado para referirme a la diferencia en las relaciones de poder que existen entre dos partes comprometidas (formal o informalmente) cuando la inversión extranjera tiene lugar. La situación en la que estoy particularmente interesado se presenta cuando una empresa transnacional invierte en un país del Tercer Mundo. Cualquier dependencia creada es el resultado de dos factores muy específicos que actualmente adquiere la economía global. El primero, consiste en la existencia de muchos lugares para establecer a bajo costo ensambladoras y fábricas para aquellas empresas transnacionales (ET) que deseen ir al exterior. Segundo, las ET grandes, e incluso las relativamente pequeñas en mercados especiali-

- Ponencia presentada en el Seminario de Investigación sobre las Relaciones México-Estados Unidos, Centro de Estudios Mexicoamericanos, Universidad de California, San Diego, abril de 1987.

* Escuela de Fconomía de Londres e investigador visitante en el Centro para Estudios Mexicoamericanos, Universidad de California, San Diego.

*.. Traducido del inglés por Guadalupe Ortega Villa. 
zados, pueden ejercer el control de la produccióni global y de la distribución en una forma que las industrias en países menos desarrollados no pueden esperar igualar. Existe un largo camino por recorrer a partir de estas bien específicas, aunque extremadamente importantes, consideraciones hacia una teoría generalizada de la dependencia del país pobre respecto del tipo de país rico. Lo que sugiero aquí es algo mucho más limitado, a fin de establecer las relaciones entre las ET y aquéllos a quienes impactan en los países menos desarrollados en los cuales invierten. Un resultado de tal relación ha sido conceptualizado por Evans (1979) como "desarrollo dependiente", y éste me parece un buen punto para empezar. Lo que planteo investigar es el potencial del desarrollo dependiente para ser revertido, proceso que ha sidollamado "reversión de la dependencia" (véanse: Doran, et. al., 1983). Se considera esto como prueba crucial de los efectos desarrollistas del capital transnacional en el Tercer Mundo, al grado en que el desarrollo progresivo sólo se llevará a cabo cuando las necesidades e intereses de la población de los países menos desarrollados comiencen a desplazar las necesidades e intereses de las ET, entonces es cuando se inicia la reversión de la dependencia y se comienza a desplazar al desarrollo dependiente. Este planteamiento inicial claramente deja colgando muchas cuestiones vitales, principalmente la lucha de clases en los países menos desarrollados y la lógica organización de la producción global del capital transnacional. Las herramientas para abordar ambos asuntos están elaboradas en la metodología que se presenta después.

Con el propósito de enfocar estas cuestiones he seleccionado cuatro casos para detallar su estudio ${ }^{1}$, sin embargo, aquí me concretaré a México. Este país tiene una larga y notable tradición revolucionaria, se ha aferrado a los problemas socioeconómicos del proteccionismo yen la última década, o antes, ha dado pasos significativos en dirección a liberalizar la economía.

México durante algún tiempo ha sido el lugar preferido para la inversión extranjera estadounidense. En tanto que los extranjeros son propietarios de alrededor del $2.5 \%$ de las firmas industriales mexicanas, más del $30 \%$ de las exportaciones industriales mexicanas derivan de compañías de propiedad extranjera, principalmente

1 Los cuatro países son Irlanda, Egipto, China y México. Los estudios de caso de los tres primeros han sido publicados o están por aparecen dentro de poco (véanse las referencias). Actualmente estoy escribiendo una monografia de la economía política de las maquiladoras. Los cuatro casos eventualmente serán el contenido de un libro en donde se evalúan los efectos en términos de desarrollo del capital trasnacional. 
compañías nortcamericanas (como lo reporta La Jomada del 18 de junio de 1985). Esto da a las trasnacionales, y particularmente a las estadounidenses, un poder de negociación considerable en asuntos del comercio exterior de México. Por tanto, la comunidad de empresarios extranjeros se convierte en un punto de referencia y esta situación puede ser denominada "intromisión extranjera a través de la inversión". Mientras más directa la inversión, de cualquier modo, más indirecta tiende a ser la intromisión. Las ET, con algunas excepciones notables, no enseñan a los gobiernos extranjeros cómo actuar, sino más bien confían en métodos sutiles para asegurar el logro de sus intereses. El más importante y penetrante de éstos se refiere al ampliamente conocido "clima para la inversión", el cual es de hecho casi un resumen del potencial de negocios para las ET. Para retener y extender la inversión extranjera directa, el gobierno anfitrión debe de asegurar que el clima para la inversión, como lo definen las ET, sea favorable, esto es, que conduzca al máximo de utilidades que sean fácilmente repatriables. Por supuesto que no discuto que los gobiernos anfitriones sean constantemente forzados a actuar en contra de sus principios debido a la presión de las ET; lo que sostengo es que las ET tienden a establecer las reglas y a marcar los límites de acción del gobierno anfitrión, particularmente en áreas cruciales como las políticas industriales y de exportación y, por tanto, de importación. No importa que los intereses de las ET y de sus agentes locales (la burgucsía compradora) frecuentemente choquen con los intereses de la clase capitalista nativa. El gobierno mexicano ha sido criticado por sectores de la burgucsía local, así como por la izquierda nacionalista, por favorecer a los industriales extranjeros por encima de los industriales de origen doméstico en la distribución de recursos cscasos (véase: Maxficl y Anzaldua, 1987).

\section{De zonas económicas a zonas de desarrollo}

En su libro Territory and function, Friedmann y Weaver demuestran lo sobresaliente que es la planeación regional para el desarrollo. Muestran cómo las doctrinas de los centros de crecimiento o polos, que capturaron la inventiva de académicos y planeadores a la par que la década de los sesenta lo demostraba, resultó en sus no disparatados términos: "completamente inútil como herramienta para el desarrollo regional" (Friedmann y Weaver, 1979, p. 175). La inversión extranjera $y / o$ ayuda externa tiene que ir a algún lugar y hacer algo, 
y desde la década de los sesenta en adelante, algo de la inversión extranjera para el desarrollo, que fluyó a los países del Tercer Mundo, fue dirigida a zonas especialmente construidas, a menudo sobre las bases de estas doctrinas inútiles. Incluso volúmenes relativamente grandes de inversión extranjera para el desarrollotuvieron leves efectos locales de "dispersión" o "escurrimiento" en las economías locales o nacionales del país anfitrión. El crecimiento de las industrias nativas subcontratantes en el "grupo de los cuatro" del este asiático, son más bien las excepciones parciales que la regla.

El estudio de lo que de manera general pueden ser llamadas "zonas económicas" me parece importante, al menos por dos razones. Primera, frecuentemente han sido las expresiones físicas de la política de desarrollo industrial en los países del Tercer Mundo. Segunda, invariablemente han sido establecidas para atraer inversión extranjera (Unido, 1971 y 1980); por tanto, tienden a concentrar los efectos del medio externo del sistema capitalista global de una forma particularmente intensa. En las "zonas productoras para la exportación" (como generalmente son llamadas) la rentabilidad de las ET que operan dentro de ellas, de hecho comúnmente depende de su integración intra-cmpresarial. De aquí que los efectos de "dispersión" o de "escurrimiento" puedan interferir con el plan empresarial, inhibir la transferencia de precios y, eventualmente, reducir las razones para tal inversión. No hay duda de que la inversión extranjera con frecuencia trae el crecimiento económico, en el sentido de incrementar el nivel de actividad, y que el comercio exterior debiera incrementarse al margen del equilibrio entre exportaciones e importaciones a medida que el volumen crezca. En lo que las zonas productoras para la exportación y las zonas libres que le son similares han fallado, salvo pocas excepciones, es en transformar este crecimiento económico en desarrollo. Con el propósito de explicar por qué éste es el caso y mostrar cómo debería ser el cambio, es necesario examinar más ampliamente el concepto de "zona económica" y relacionarlo con los problemas del desarrollo. Para hacer esto propongo el concepto de "zona de desarrollo" y especificar cómo en la práctica debería funcionar para producir el crecimiento económico y el desarrollo; esto con la intención de hacer una contribución concreta a la política actual para el desarrollo, así como una contribución teórica a los planteamientos en torno al desarrollo y al subdesarrollo.

Las zonas, sin embargo, no están completamente aisladas de los medios interno y global. Existen dos temas amplios específicos 
acerca de la inversión extranjera para el desarrollo en el Tercer Mundo que repercuten de manera importante en las zonas económicas, y son: a) género y, b) la "triple alianza". El género considera los efectos que la inversión extranjera tiene a través de la nueva división internacional del trabajo en las estructuras patriarcales que dominan la mayor parte de los países del Tercer Mundo, y en ocasiones a la inversa. El proceso de exportación y las industrias ensambladoras que tienden a ser predominantes en las zonas económicas, como es bien sabido, principalmente contratan a mujeres jóvenes, aunque la mayoría de la literatura sobre el tema tan sólo registra los hechos y fallan al tratar de averiguar su significado en forma detallada (véase por ejemplo: Froebel, et al. 1980). Sin embargo, un número creciente de teorías feministas acerca del desarrollo así como de investigaciones están retando esta negligencia, y ahora existe una buena cantidad de evidencia para opinar que, lejos de ser un producto, la división sexual del trabajo y las cambiantes relaciones patriarcales son componentes fundamentales de esta nueva división internacional del trabajo. En México, la preponderancia de las mujerestrabajadoras en las maquilas hace que estas últimas se parezcan mucho más a las zonas económicas del tipo tradicional que producen para la exportación; entonces, con la nueva división internacional del trabajo, ambas producen una nueva división sexual del trabajo y reproducen una división sexual tradicional del trabajo en una variedad de formas frecuentemente contradictorias (Véase: Lim, 1985). Por tanto, la creación de zonas de desarrollo y la expectativa de desarrollo en sí, en algunos casos puede articularse a las formas en que cambiarán las relaciones sociales patriarcales, particularmente aquéllas en torno a la división sexual del trabajo. En este ámbito existe un potencial importante para el conflicto entre las fuerzas que promueven la inversión extranjera y aquéllas que la resisten.

El segundo tema, el de la "triple alianza" (o en los términos de Evans, alianza tripartita) de las ET, la burocracia del país anfitrión y la burguesía local compradora hacen mucho más específicas las cuestiones clasistas acerca de quién exactamente se beneficia con la inversión extranjera y quién paga los costos. Richard Sklar ha adelantado algunos argumentos de peso en contra de la existencia de una burguesía compradora, y ha propuesto sustituir el término por el de "burguesía gerencial" y evitar la insinuación de que esta clase carece de un nacionalismo genuino (Sklar, 1976). Para aclarar este punto, es importante reconocer que en este contexto el "comprador" implica 
la existencia de una fracción de la burguesía nacional que considera que la mejor forma de servir a los intereses nacionales es el de la alianza con el exterior, que generalmente son fuerzas occidentalizadoras. El nacionalismo es una ideología particularmente elástica. Los partidarios locales de la triple alianza, en la mayoría de los países del Tercer Mundo, son generalmente aquéllos que salen ganando, mientras que los verdaderos representantes de los trabajadoras generalmente brillan por su ausencia. Esta es una cuestión eminentemente a investigar, para la cual México proporciona fuentes ricas en información y en expectativas teóricas.

Estas cuestiones generales de género y clase, entonces proporcionan un contexto en el cual las cuestiones específicas pueden desarrollarse. Con el proposito de hacer esto se proponen seis criterios con los cuales se puede medir el grado en que una punto se convierte en zona de desarrollo. Estos criterios proporcionan a corto plazo una prueba de la posibilidad de reversión de la dependencia bajo las condiciones actualmente existentes de género y clase en cualquier sociedad.

\section{Criterios para la transición de zonas económicas a zonas de desarrollo}

1) Eslabonamientos concretos son la participación de las importaciones (hacia atrás) y la participación de las exportaciones (hacia adelante) en la producción de una empresa que viene y va a la economía receptora. Una guía preliminar es que a mayor extensión de los lazos hacia atrás (materias, componentes, servicios) y a mayor extensión de los lazos hacia adelante (ventas a las industrias de bienes intermedios) que sc obtengan con la economía receptora, más sc asemejará a la creación de una zona de desarrollo (las "exportaciones" de bienes de consumo acabados desde las zonas hacia la economía anfitriona son excluidos enfáticamente de la categoría de eslabonamientos hacia adelante como son usados aquí).

2) A mayor valor agregado en el país receptor y a mayor proporción de divisas retenidas en la economía receptora (referida de diversos modos complejos), traerá como consecuencia una mayor similitud con la creación de una zona de desarrollo.

3) A menor proporción de desnaturalización de los gerentes locales, técnicos y personal altamente calificado, se asemejará más a la creación de una zona de desarrollo.

4) A mayor grado de transferencia de la tecnología local (lo que 
preferiría llamar, siguiendo a Adikibi, comotecnología sujeta), mayor será el parecido a la creación de una zona de desarrollo.

5) Cuanto más favorables sean las condiciones diarias de trabajo para la fuerza de trabajo en relación a las condiciones prevalecientes en el resto de la sociedad receptora, más se asemejará a la creación de una zona de desarrollo.

6) Cuanto más equitativa sea la distribución de los costos y de los beneficios entre los inversionistas extranjeros, así como la competencia para escalar un estrato entre los grupos de la población local y el gobierno anfitrión en la zona, más se asemejará a la creación de una zona de desarrollo.

Estos scis critcrios claborados para estudiar la cvolución de una auténtica zona de desarrollo no son condiciones de todo o nada, pero continúan constantemente en movimiento. Los eslabonamientos, la retención de divisas, el ascenso del personal local, la tecnología, las condiciones de trabajo y de distribucion de los beneficios, por tanto, pueden tener valores positivos o negativos como consecuencia de las políticas que son llevadas a cabo para producir el desarrollo regional. En el peor de los casos, existen eslabonamientos hacia atrás y/o hacia adclante que quizá sean destruidos como resultado de la creación de una zona económica; y similarmente, la retención local de divisas puede declinar, se pueden perder habilidades locales, la explotación de la fuerza de trabajo se puede intensificar, y por tanto, la distribución puede llegar a ser más inequitativa que antes. En mis términos esto significaría que no hay zonas de desarrollo en el proceso de creación, sino importa qué tanto "crecimiento económico" hubiera (por ejemplo, el incremento del PNB per cápita o el incremento del comercio cxterior), y por tanto, no desarrollo. Pronosticaría que bajo tales circunstancias los problemas del desarrollo en la región en cuestion, y por implicación en todo el país, se incrementarían.

El caso neutral, cuando hay un ligero cambio en los criterios, significa que puede existir el potencial para la evolución de una zona de desarrollo, pero los cambios en las políticas son necesarios para poner de manifiesto este potencial. El caso positivo, en donde todos los criterios se están fomentando, habla por sí mismo. Evidentemente, los criterios pueden cambiar en diferentes proporciones en el tiempo y en el espacio, y los problemas sustantivos que esta teoría omite, principalmente involucran tales cuestiones.

Aunque aquí no puede argumentarse detenidamente el caso, la manera más fructífera para analizar estos criterios es en términos de sus efectos económicos, políticos y culturales. He empezado a hacer esto en mi estudio de las ET en la república de Irlanda (Sklair, por 
aparecer b). Tal métodode análisis evidentemente implica el potencial para el logro de una limitada reversión de la dependencia. Permítanme considerar el caso de las maquiladoras en México.

\section{Las maquiladoras en México. ${ }^{2}$}

A mediados de la década de los sesenta el gobierno mexicano introdujo un Programa de Industrialización Fronteriza (PIF), la plataforma más grande que se instalo para permitir que las fábricas de propiedad extranjera operaran libres de impuestos a lo largo de la frontera (por eso comúnmente es llamada industria "in-bond"). Estas fábricas, principalmente de propiedad estadounidense, también eran capaces de tomar ventaja de las reglas arancelarias de ese país, que cubren la reimportación de bienes ensamblados sin terminar que usan componentes manufacturados en Estados Unidos. Estos eran los principios del programa de maquiladoras. Entonces, ¿qué puede decirnos la metodología propuesta sobre Méxicoy la relevancia global de desarrollo de la estrategia maquiladora? En términos generales, la idea de permitir la inversión extranjera basada en fábricas que en su totalidad son propiedad extranjera, localizadas en o cerca de parques industriales privados, en lugar de inversiones conjuntas en zonas planeadas o relativamente amplias, está sujeta a fomentar cierto tipo de desarrollo. Es verdad que México tiene actualmente entre 800 y 1000 maquiladoras que dan empleo a un promedio de 250,000 a 300,000 trabajadores, y que los ingresos por exportaciones en la región fucron del orden de $\$ 1,500$ millones de dólares para 1986. Esta es una situación de éxito genuino y no una exageración para describir a la industria maquiladora como un "boom" a lo largo de la frontera y en el interior, en donde ahora también se permiten las maquiladoras. Sólo se necesita observar las docenas de firmas de consultorías y trámites que se han esparcido en México y en los Estados Unidos durante los últimos años para confirmar esta impresión.

Sin embargo, en términos de los seis criterios, ¿podemos afirmar que las principales plazas maquiladoras efectivamente se han transfor-

${ }^{2}$ La evidencia a seguir es muy grande en mis apuntes del trabajo de campo, los cuales están en proceso de ser escritos. Una buena narración reciente acerca de las maquiladoras se encuentra en el capítulo de Grunwald en: Gibsony Corona Rentería, eds. 1985; en sí todo el libro es una fuente excelente; para los fundamentos legales véase: Gritsch 1986; y una encuesta reciente de las maquiladoras en Baja California y su impacto en los Estados Unidos (Clement y Jenner, 1987) contiene mucha información de utilidad. Las fuentes básicas son los datos de las series del INEGI y la revista mensual: "Maquiladora Newsletter" en la Cámara Americana de Comercio en México 
mado en zonas de desarrollo? La investigación efectuada se ha centrado en maquiladoras de Tijuana, Mexicali, Ciudad Juárez, Reynosa y Matamoros, cubriendo aproximadamente el $75 \%$ del total en México.

Los eslabonamientos son muy escasos, alrededor de 1-2\% del total de compras; por tanto las fábricas maquiladoras no contribuyen en concreto al crecimiento de la industria local, o de la industria en cualquier otro lugar en México; de aquí que existan tres dimensiones en este problema, debido a que la lógica de la producción transnacional al mismo tiempo fomenta, permite o prohibe los lazos hacia atrás; donde un producto es completo y horizontalmente integrado dentro de las ET o es un abastecedor cautivo del sistema, o donde los componentes intermedios y/o materiales usados son de naturaleza tan especializada que simplemente no hay abastecedores disponibles fuera del sistema de la ET existente, entonces los lazos hacia atrás están literalmente prohibidos por la lógica global de la producción. En el estudio sobre Irlanda, en donde hay muchas ET altamente especializadas que producen para el mercado mundial, hay varios ejemplos de esto (Sklair por aparecer b, 3.6).

Sin embargo, la mayoría de las ET que operan en localidades con bajos costos tienden a estar involucradas en líneas de producción más tradicionales tecnológicamente rezagadas, donde los materiales y los componentes tienden a estar más prontamente disponibles. Este es el caso donde, al menos superficialmente, la lógica de la producción de las ET parece permitir eslabonamientos hacia atrás. Ciertamente este es el caso de México, donde muchas maquiladoras de propiedad estadounidense, por ejemplo, demandan estar en busca de materiales y componentes locales. Además, las autoridades mexicanas a nivel federal, local y paraestatal, por varios años han tratado de organizar laindustria mexicana para surtir a las maquiladoras de lo que necesitan. Propiamente, esto es visto como una bonanza potencial para la industria mexicana. Generalmente, las razones por las cuales esto no ha sucedido son mencionadas como: a) la producción mexicana no es de la calidad requerida por el mercado mundial;b) los precios son muy altos; y c) la entrega no es confiable. Nadie que esté familiarizado con la actuación de la industria doméstica mexicana se asombraría de ninguna de éstas, pero aún así uno puede preguntarse por qué la imagen para las fuentes locales es tan baja. Esto sugiere que la lógica global de producción (como la llamo) está operando de nuevo, pero de una manera diferente de la arriba mencionada. Aquí las cuestiones de transferencias de precios y abastecedores cautivos se tornan cruciales, pero debido a la naturaleza del caso, esto actualmente es casi 
imposible de investigar en México.Sólocuandolas fábricas mexicanas, de hecho empiecen a producir a precios competitivos lo que las maquiladoras necesitan, la calidad y la entrega estarán en posibilidad de contestar estas preguntas. Esto puede llevar algo de tiempo, pero ciertamente debe de ser incluido en la agenda para las futuras evaluaciones de la estrategia para la maquila. Hay pocos casos en los cuales los lazos hacia atrás han sido activamente fomentados, eso es cuando una maquila ha ido en busca de materiales o componentes en las agencias o manufacturas locales. Aquí el problema para México es que este contexto "local" generalmente significa ambos lados de la frontera y en algunas áreas, evidentemente el sur de California, son más bien las pequeñas firmas estadounidenses y no las mexicanas las que aprovechan estas oportunidades dadas para los abastecedores locales.

En una industria exclusivamente orientada a la exportación, los eslabonamientos hacia adelante con la economía anfitriona no surgirían. Sin embargo, es claro que los días en que sólo se procesaba para la exportación están contados, precisamante por la manera en que evoluciona la economíaglobal. En México, las decisiones recientes que permiten que las maquilas se vendan unas a otras y que permiten el acceso limitado al mercado interno como un aliciente para el abastecimiento local, al mismo tiempo que no producen de inmediato una actividad abundante, evidencian que la producción para la exportación no excluye tanto efectos de los eslabonamientos hacia delante como hacia atrás en la economía doméstica local. Por tanto, no hay nada esencial que impida que las maquilas den abasto de bienes intermedios a los productores mexicanos, y no hay duda de que esto puede hacerse beneficiosamente para ambas partes. En Matamoros, por ejemplo, una maquiladora cuyos propietarios son de Illinois ya está vendiendo filtros electrónicos a firmas mexicanas. El punto aquíes que no es simplemente la capacidad de la economía anfitriona para atender los requerimientos de los lazos, que es importante, sino que la lógica de la producción global permita o prohiba tales eslabonamientos en desafío de las condiciones económicas locales.

$\mathrm{El}$ asunto de las entradas de divisas, de las ataduras de la estrategia de la IEAIET, tienen una dimensión simple y una compleja. La dimensión simple se refiere al valor monetario del valor agregado en divisas debido a la producción de la maquila. La aritmética puede ser muy complicada debido a la necesidad de incluir las importaciones requeridas para producir las exportaciones, los aranceles donde son 
aplicables y el valor relativo de las divisas; sin embargo, no es imposible llegar a una cifra razonablemente certera. De hecho, las autoridades mexicanas en estadística han claborado cifras para establecer los ingresos de divisas producidas por las maquilas para muchos años atrás, y recientemente esta cantidad ha desplazado al turismo del segundo lugar como actividad generadora de divisas para México, detrás de las exportaciones de petróleo. Con eslabonamientos casi inexistentes el valor agregado de la maquila, por tanto, está casi exclusivamente en términos de salarios de la fuerza de trabajo empleada, de la ganancia y de otros ingresos. La dimensión compleja del asunto no es qué tantas divisas son captadas, sino qué tantas son retenidas en México. La proporción de dólares retenidos en México varía dramáticamente con el valor del pesoy de los patrones de gasto de la fuerza de trabajo. Varios estudios han sugerido que antes de la devaluación del peso en 1982 y aún después, los trabajadores de las maquiladoras gastaban más de la mitad de sus salarios en los Estados Unidos, en las tiendas y centros comerciales de los poblados estadounidenses en la frontera. En 1987, cuando la paridad peso-dolar era 1000 a 1 deslizándose diariamente, todavía algunos denunciaban que la mitad de los salarios provenientes de las maquiladoras eran gastados en Estados Unidos. Por supuesto, esto significa que la retención de dólares dentro de la economía mexicana está menos distante que el valor agregado debido a la industria maquiladora. Debemos, por tanto, examinar las relaciones entre los ingresos por la exportación de la maquila, los costos de importación de la misma, la retención de dólares y la paridad del peso respecto al dólar.

Existe una gran cantidad de información acerca del personal mexicano con altos puestos. El campo de investigación a lo largo de la frontera confirma las declaraciones de que los nacionales mexicanos habitualmente retienen posiciones administrativas y responsabilidades técnicas en las maquiladoras. En tanto que la mayoría de las maquilas continúan ubicando en los puestos cumbre a ciudadanos norteamericanos, hay algunos mexicanos que manejan sus propias maquiladoras. No hay duda de que el estilo trasnacional técnico y administrativo son trasmitidos rápidamente a un gran número de mexicanos y ya hay evidencias de que este estilo está penetrando a todos los niveles de la industria mexicana. Dadas las quejas comunes de que la industria interna mexicana ha estado protegida por tanto tiempo que noes posible afrontar la competencia global, el significado de este hecho no debiera ser subestimado. Por otra parte, no se puede 
suponer que los técnicos y cjecutivos mexicanos se identificarán más fácilmente con los intereses y objetivos nacionales mexicanos y chocar con los intereses y metas de los empresas que les dan empleo, que como lo harían los ejecutivos extranjeros. La creación de una burguesía compradora, como he sostenido, puede tener consecuencias contradictorias para el desarrollo.

Aparentemente, la transferencia tecnológica se está llevando a cabo en todas los lugares de México. Sin embargo, el anclaje tecnológico, salvo pocas excepciones, es muy poco visible. Es importante distinguir entre, por ejemplo, la General Motors que produce partes para automóvil en una planta de la General Motors (ya sea en Detroit o en Matamoros), y una General Motors que produce partes para automóvil en una planta en los Estados Unidos o en una maquiladora mexicana. Esta expresión más bien enredosa es mi forma de argumentar que existe una diferencia entre la GM que produce partes completamente bajo el control de la GM en cualquier planta GM dondequiera que se localice, y una planta mexicana local que produce partes para automóvil, ya sea para la GM o cualquier otra empresa. El primero es reubicación de tecnología (con frecuencia llamado equivocadamente transferencia de tecnología), el último es anclaje de tecnología. En las maquiladoras hay muchas pruebas de reubicación de tecnología pero muy pocas de anclaje tecnológico. Sin embargo, en algunos sectores de electrónica, como la manufactura de placas de circuitos impresos, y en la fabricación de partes para automóvil, ciertamente se está efectuando algo más que el simple ensamble.

No debe sorprendernos que las condiciones laborales en las maquiladoras dependan del tipo de fábrica de que se trate, del lugar donde se encuentran, y con qué se les está comparando. Ha existido una gran cantidad de literatura acerca de la explotación de los trabajadores, especialmente de las mujeres trabajadoras, en las maquilas. Rara vez queda claro que ya sea que entendamos esto en términos de "superexplotación" de los trabajadores en las maquilas o en el sólo hecho de que la propiedad extranjera constituya una explotación en sí misma, o bien en que las industrias en las cuales las maquiladoras están típicamente concentradas tiendan a ser más explotadoras que otras industrias mexicanas; mi posición al respecto es que todo el capital es explotador, en tanto que expropia el plusvalor de los trabajadores que lo están creando. La nacionalidad del capital por sí misma hace una diferencia bajo ciertas circunstan- 
cias, pero no todas, y es importante notar que la estructura de la industria de propietarios extranjeros sí difiere de aquella cuyos propietarios son locales, y esto puede hacer una diferencia en el grado de explotación si optamos por medirlo en un sentido o en el otro.

Debemos identificar al menos cuatrocondiciones diarias de trabajo que son relevantes en este contexto. Por tanto, aunque todos los trabajadores bajo relaciones capitalistas de producción sean explotados, podemos medir el grado de explotación que sufre un grupo de trabajadores comparado con otros grupos de trabajadores en términos de sus salarios respectivos, seguridad en el trabajo, horas trabajadas, y prestaciones laborables. No hay duda de que los trabajadores de las maquiladoras mexicanas (y ejecutivos por ese solo hecho) son altamente explotados comparados con los trabajadores (y ejecutivos) en una fábrica similar en los Estados Unidos. Ciertamente, ésta es una medida bastante válida y es una interpretación significativa de la explotación. Otra medida significativa y válida de la explotación es comparar a los trabajadores de la maquila con los trabajadores de la industria propiedad de nacionales, ambos en la región fronteriza o en cualquier otro lugar. En estos casos existe una gran cantidad de evidencia que sugiere que los trabajadores de las maquilas obtienen mejores salarios que los trabajadores no-maquiladores (efectivamente, el nivel del salario mínimo federal es más alto en las zonas fronterizas, donde se ubican la mayoría de las maquiladoras, que en cualquier otrolugar de México). La seguridad en el empleo hace surgir algunos temas muy interesantes, precisamente debido a que las maquiladoras con frecuencia son etiquetadas como "industrias golondrina", y a que ha habido varios casos notorios de maquiladoras norteamericanas que silenciosamente se van durante la noche para evadir el pago de las indemnizaciones a los trabajadores mexicanos (véase: Baird y McCaughnan, 1979). Durante la recesión norteamericana, después del golpe petrolero y nuevamente después en 19811982 , muchas maquiladoras cerraron. Noobstante, la investigación que se lleva a cabo indica que, contrariamente a esta pobre imagen de la industria, las plantas maquiladoras en un sentido amplio, ya no fácilmente cierran industria por industria, sino que actúan como cualquicr otra planta ubicada en cualquier lugar de México o de los Estados Unidos. El hecho es que debido a las maquiladoras existen ahora más de 150,000 empleos nuevos en las industrias manufactureras en México que no existían en 1982, cuando el peso inició su decisivo deslizamiento.

Similarmente, en horas trabajadas a la semana no hay diferencias 
sustanciales entre la industria maquiladora y el conjunto de la industria mexicana. De hecho, el único conflicto sindical exitoso en México por conseguir las 40 horas de trabajo a la semana se llev6 a cabo entre los trabajadores de maquiladoras en Matamoros (Es interesante advertir que a las maquiladoras que se establecieron en Matamoros después de haber ganado la semana laboral de $\mathbf{4 0}$ horas, se les concedió un permiso por cinco años para laborar 48 horas a la semana y posteriormente establecer la semana laboral de 40 horas).

Las prestaciones laborales, por las cuales entiendo salud, disposiciones de seguridad, alimentos subsidiados, recreación, etc.; tienden a ser una función que depende del tamaño de la planta más que de su propietario. El número promedio de empleados por maquila está por encima de $\operatorname{los} 250$, más del doble de la industria mexicana en conjunto, lo que sugiere que los trabajadores de las maquiladoras no están más mal que otros trabajadores mexicanos. Esto no significa que todas las maquiladoras sean lugares salubres y seguros, existen varios estudios que documentan la salubridad y los riesgos en la industria (véase por ejemplo: Carrillo y Jasis, 1983).

Una cuestión central para la discusión de las condiciones de trabajo son la representación de los trabajadores y la acción. La industria maquiladora está sindicalizada casi en un cien por ciento en Matamoros y Reynosa, donde la CTM las controla, pero el nivel de sindicalización declina rápidamente en la medida en que nos encaminemos rumbo Oeste, y en Tijuana no hay movimiento organizado efectivo en la industria maquiladora. Sin embargo, como ha demostrado Carrillo(1985), las protestas laborales, las huelgas y las demandas laborales, han tendido a ser más numerosas donde la participación sindical es más baja que en aquellas en donde es más alta. La rotación laboral es también mucho más alta de Juárez haciael Oeste que del lado Este de la frontera, aunque es incierto saber si es una simple función del escaso mercado de trabajo o del escaso control sindical.

Lo cierto es que los trabajadores de las maquiladoras, como los trabajadores en cualquier parte de México, han sufrido un descenso en sus estándares reales de vida durante los últimos años, mientras que las ganancias de las ET se han incrementado sustancialmente e incluso más vívidamente, cada caìda del peso respecto al dólar parece anunciar una nueva ola de establecimientos de maquilas. El deslizar el peso respecto al dólar ha golpeado a los trabajadores de las maquiladoras en la frontera de una forma particularmente fuerte, porque las comunidades fronterizas mexicanas siempre han buscado 
en los Estados Unidos cuando menos algunos de sus satisfactores esenciales, y muchos de los no esenciales. En general, los consumidores fronterizos no han dispuesto de la alternativa de los productos mexicanos, o ha sido muy cara, o de inferior calidad, o disfrazada como importaciones norteamericanas. Sin embargo, como lo indica una investigación reciente en Ciudad Juárez, no sería cierto decir que los trabajadores de las maquilas son los grupos más pobres en sus localidades o nacionalmente hablando. Por supuesto, esto es una cuestión muy compleja e involucra no sólo a los trabajadores de la maquila sino a su familia, que a menudo se encuentra en ambos lados de la frontera. También es de importancia notar que los profesionistas mexicanos y la burguesía compradora a lo largo de la frontera, generalmente se han comportado muy bien fuera de los desarrollos de parques industriales para maquiladoras, y los servicios legales y comerciales para la industria maquiladora han hecho de estos agentes una nueva clase de ricos mexicanos (y por supuesto, también de los agentes estadounidenses). La distribución de los costos y las utilidades siempre será extremadamente difícil de determinar con cierto grado de exactitud, aquí se quisiera indicar lo que debería ser tomado en cuenta en el caso de las maquiladoras. Las cuestiones de las condiciones de trabajo y distribución en la industria de la maquilas surgen inmediatamente, además de las cuestiones de género, de la división sexual del trabajoy de la división sexual del trabajo doméstico. Existe ahora una gran cantidad de literatura acerca de las "mujeres en las maquiladoras", mucha de ésta proclama la velada suposición de que de alguna manera, los trabajos para las mujeres jóvenes son menos valorados que los trabajos para los hombres y el hecho de que la maquila emplea mayoritariamente a mujeres, esto es en sí una crítica válida acerca de la industria. Estas por supuesto son posturas completamente sexistas e ignoran la participación de la mujer en la industria, establecida en varios países desde hace muchos años incluyendo a México, como lo ha argumentado decisivamente Susan Tiano (En: Ruiz y Tiano, por aparecer). Por otro lado, es importante reconocer que los empleadores de la maquila se caracterizan por preferir emplcadas femeninas, debido a que las mujeres trabajadoras tienden a ser más dóciles y menos propensas a organizarse que los empleados hombres. Es también significativo que la proporción de empleados varones en las maquiladoras se ha estado incrementando firmemente en los últimos años, particularmente en Ciudad Juárez, donde la mayoría de las contrataciones recientes 
se dice son hombres. Las razones de esto son inciertas, pero es probable que la demanda de mujeres trabajadoras para la maquila en y alrededor de Cd. Juárez haya sobrepasado la oferta. Una situación bastante diferente existe en Tamaulipas (contigua al sur de Texas), donde actualmente hay una oferta abundante de fuerza de trabajo, y las plantas maquiladoras están casi totalmente sindicalizadas.

No hay duda de que los problemas de género inciden seriamente en los problemas de distribución en donde el desempleo masculino es significativamente mayor que el femenino, $y$ en donde las mujeres parecen estar ocupando puestos para los cuales los hombres están preparados y que descan ocupar. Existe algún indicio en las investigaciones de las maquilas de que algo así está sucediendo en esta industria. Los temas fundamentales surgidos por tales circunstancias son generalmente ignorados por los teóricos del desarrolloy, de nuevo señalo que deben ser considerados en la agenda para futuras investigaciones.

\section{Maquiladoras para México y maquiladoras para los Estados Unidos ${ }^{3}$}

En diciembre de 1986 sucedió algo poco común. El Departamento de Comercio estadounidense fue forzado, claramente en contra de su voluntad, a retirar su patrocinio a la Expo-Maquila 1986, una muestra comercial diseñada para exhibir las ventajas de laș maquiladoras a los manufactureros estadounidenses. La oposición para que se involucrara el Departamento de Comercio en la Expo vino principalmente de los políticos de las zonas industriales tradicionales, preocupados por la pérdida de empleo sufrida por sus representados. Por este motivo la AFL-CIO a partir de la década de los sesenta, cuando se introdujo el PIF, siempre ha estado haciendo campaña en contra de las maquiladoras, aunque la oposición sindical siempre ha sido más bien en tono bajo, fue consistente y los mismos temas han surgido una y otra vez. La industria maquiladora nunca ha estado organizada políticamente de manera sistemática, aunque está la CANACINTRA que vincula la Asociación Mexicana de Maquiladoras(Consejo Nacionalde la Industria Maquiladora de Exportación)

${ }^{3}$ Esta sección está basada en material confidencial recopilado a lo largo de la frontera, complementado por artículos de los periódicos locales y de la edición de enero de 1987 de la Asociación de Comercio Exterior de El Paso "Paso del Norte trade News". 
con una organización nacional que guarda un perfil tenue y que presenta pocas evidencias de que haya tenido muchos efectos en la evolución de de la industria. Esta asociación publica una revista vistosa llamada "Ensamblador", y en algunos lugares (evidentemente Cd. Juárez) la asociación local efectúa investigaciones útiles acerca de la industria. Por tanto, noes muy sorprendente que durante este último ataque a las maquiladoras surgido en Washington, fueran los intereses norteamericanos y no los intereses mexicanos los que inmediatamente salieran a la defensa de la industria maquiladora.

Después de pocas semanas de retirado el patrocinio del Departamento de Comercio a la Expo (el cual, a propósito, continuó bajo el patrocinio privado y fue de todas cuentas bastante exitoso en términos de promoción), los influyentes de las maquiladoras se reunieron en El Paso para planear una estrategia, ya que percibieron la llegada de lo que sería la disputa por la legislación sobre comercio y aranceles que podría suprimir efectivamente la industria maquiladora. ${ }^{4}$ Más de 150 personas asistieron a la reunión de El Paso, oficialmente conocida como la Conferencia para la Estrategia del Comercio Fronterizo, que incluyó aproximadamente 50 delegados representantes a las ciudades fronterizas desde San Diego/Tijuana en el oeste hasta Brownsville/ Matamoros en el este. Si bien es difícil probarlo, creo que la campaña para defender las maquiladoras fue de hecho concebida en Washington por el equipo de políticos de los estados fronterizos y sus seguidores, en respuesta a las presiones de los intereses de las maquiladoras de sus estados. Aunque los gerentes de las maquiladoras pronto se vieron involucrados, ellos no iniciaron la campaña yesto puede ser explicado por el hechode que la industria maquiladora generalmente ha evitado la publicidad, excepto aquella de los promotores comerciales -el grupo que catalogo como"gestores"-. A principios de 1987 la campaña había desarrollado una forma de organización como la Alianza Comercial Fronteriza (ACF), y los gestores viajaban a lo largo de las ciudades y poblados fronterizos norteamericanos tratando de organizar a la industria maquiladora (tareaque fue másfácil en algunoslugares que enotros), para afrontar el ataque, ésos fueron impulsos provechosos. Para marzo, cuando

4 Aunque la lucha del congreso se ha centrado en torno a las regulaciones arancelarias estadounidenses $(806 / 807)$ que permiten la reimportación de bienes ensamblados con componentes manufacturados en Estados Unidos, etc., no es de ninguna manera cierto que la abolición de estos artículos afectaría a muchas maquiladoras al grado de que provoque su clausura. 
la ACF puso en Washington su más grande esfuerzo en gestoría para la defensa del programa maquilador, la mayoría de las ciudades y poblados fronterizos norteamericanos habían preparado escritos en donde exponían su opinión, tratando de demostrar que las maquiladoras significaban empleos, ingresos y negocios no sólo en México sino en los Estados Unidos. Por tanto, es una rara ironía que los propios intereses estadounidenses en la industria maquiladora proveyeran la evidencia que nos permite determinar la distribución de los costos y beneficios de la industria.

El razonamiento principal en cl argumento de la ACF es que lejos de causar pérdida de empleos en los Estados Unidos, las maquiladoras en realidad conservan e incluso crean empleos en Estados Unidos. La razón que está detrás de estas demandas esfalsa y simple, en cuanto está basada en la suposición sin argumentos de que el nivel de costos del trabajo estadounidense provoca la búsqueda inevitable de fuerza de trabajo más barata. Esto cae en la noción ideológica de "producción compartida", que significa que las empresas estadounidenses concentrarán sus operaciones intensivas en trabajo en localidades del Tercer Mundo donde la fuerza de trabajo es barata, mientras que retienen las operaciones intensivas de capital/conocimiento en los Estados Unidos. En estas premisas, las maquiladoras (y las operaciones de tipo maquilador en cualquier parte del mundo) ayudan a mantener la industria manufacturera estadounidense en condiciones de competitividad y por tanto conservan los empleos en Estados Unidos. No sólo esto, sino que las maquiladoras mexicanas consumen principalmente componentes y materiales fabricados en Estados Unidos, conservando aún más empleos en ese país que se habrían perdido si los manufactureros norteamericanos se fueran al lejano oriente. Además, la población fronteriza mexicana hace gran parte de sus compras en los Estados Unidos, y por eso, hasta una parte de los sueldos de la maquila regresa a cse país.

Scría absurdo esperar que la ACF hiciera un análisis de economía política de la industria maquiladora, por tanto, trataré de hacerlo en su lugar. Es desde luego cierto que cualquier ejemplo dado de la "producción compartida" es inevitable, aunque se pudiera probar globalmente que es un proceso irreversible (asunto en el cual me declaro no creyente). Por ejemplo, un caso reciente que provocó una atención considerable por parte de los medios de comunicación en Búfalo, Nueva York y Brownsville, Texas: se refería a un manufacturero norteamericano, de hojas limpiadoras, quien decidió cerrar la 
producción en Búfalo y transferir la mayoría de los empleos del piso de compras a una maquiladora en Matamoros. Un estudio local en Cornell muestra que si la compañía hubiera reinvertido capital para mejorar la tecnología en su planta de Búfalo, podría haberse mantenido competitiva y conservar los empleosen Búfalo (Lazes 1986). Hay, por supuesto, millones de trabajadores manufactureros norteamericanos que compiten muy satisfactoriamente con las importaciones extranjeras producidas por trabajadores cuyos ingresos distan mucho de los de ellos. Quizá la conclusión adecuada para esta corriente de argumentos es que las maquiladoras son un sostén para las industrias norteamericanas ineficientes, particularmente aquéllas con sueldos altos, altamente sindicalizadas, en áreas decadentes y que las maquiladoras son más exactamente conceptualizadas como una extensión del "cinturón del sol"s estadounidense que casualmente se localizan al cruzar la frontera en México.

Tambíen es importante comprender quién gana y quién pierde en Estados Unidos con las maquiladoras. Claramente, la clase trabajadora manual en la "zona decadente"t6 pierde empleos que son difíciles, si no imposibles, de recuperar; la creación de empleos que surgen por las maquiladoras, incluso en las zonas decadentes, tenderán a ser intensivas en tecnología aunque unos pocos de sus hijos podrían beneficiarse, el desempleo en las zonas decadentes parece constituir una generación perdida. Igualmente, existe poca evidencia de que muchos de los empleos manufactureros son creados por las maquiladoras en las comunidades fronteriza norteamericanas como consultorías profesionales de negocios, abogados, agentes aduanales, compañías de bienes raíces, vendedores al menudeo, hoteles, empresas de renta de automóviles y hasta algunos académicos, particularmente en El Paso y San Diego se están conduciendo bien fuera de las maquiladoras; sin embargo, las "plantas gemelas" manufactureras existentes en el lado estadounidense son muy escasas. La ACF reúne algunos argumentos y datos poderosos para demostrar que las maquiladoras hacen bastante por los Estados Unidos, particularmente, como lo he sostenido, grupos específicos a lo largo de la frontera. Los beneficios para México, aparte del valor real de más de 300,000 empleos industriales y algunos ingresos de operación al

5 N.del T. "sunbelt" en el documento original.

${ }^{6} \mathrm{~N}$. del T. "rustbelt" en el documento original. 
estadoy a los promotores privados, no se discuten por la sencilla razón de que la ausencia de beneficios para Méxicōes la imagen que refleja la presencia de beneficios para Estados Unidos ( $y$ viceversa, si alguna vez se logra).

Esto, por supuesto, redondea el argumento de los seis criterios para la creación de zonas de desarrollo. El que la ACF haya montado tan poderosa defensa de las maquiladoras muestra que la distribución actual de costos y utilidades no ha tallado en la piedra. La ACF debe de estar mirando de reojo al gobierno mexicano, aunque la mayor parte de su atención ahora haya sido dirigida a los legisladores en Washington.

\section{CONCLUSIONES}

Quiero ser el primero en admitir que esta conclusión es bastante inconcluyente. La investigación que he efectuado ha pretendido ensayar una metodología para determinar la probabilidad de reversión de la dependencia, que es el potencial para transformar las zonas económicas en zonas de desarrollo. Para las principales plazas maquiladoras en México he sugerido que aún necesitamos mantener la mente abierta en los acontecimientos, y se ha tratado de mostrar cómo la lógica de la producción transnacional y los preceptos de una estrategia progresiva de desarrollo pueden entrar en conflicto en puntos clave. El desarrollo, como el crecimiento económico, si es que va a suceder, tiene que presentarse en determinados lugares y en determinados momentos. La gran mayoría de los gobiernos del Tercer Mundo tienen políticas de puertas abiertas a una u otra clase de intromisión extranjera, ya sea a través de la inversión directa, ayuda o colaboración; generalmente se ha considerado que es necesaria para que el crecimiento económico se lleve a cabo. He sugerido que un eslabón entre el crecimiento económico y el desarrollo es forjado y/ o roto en la lucha entre aquéllos que promueven los intereses del capital transnacional y aquéllos que promueven los intereses de la población anfitriona. Estos intereses pueden coincidir a corto plazo, pero existe un conjunto creciente de evidencias que sugieren que a largo plazo son fundamentalemnte antagónicos. La zona de desarrollo, sostengo, proporciona la ubicación más realista para el crecimiento económico, y los seis criterios para la creción de una zona de desarrollo exitosa identifican el proceso concreto que implica la transformación del crecimiento económico en desarrollo. 
El significado de las zonas de desarrollo, como se caracteriza aquí, es reconocer la ambigüedad de una realidad en la cual los procesos de dependencia pueden ser confrontados con procesos de desarrollo. Estos retos, sin embargo, no sólo se están llevando a cabo en las discusiones téoricas abstractas, sino también en localidades concretas, como en la frontera México-Estados Unidos. Incluso en zonas potenciales de desarrollo, los soportes internos y externos de la dependencia y los obstáculos internos y externos al desarrollo son sustanciales. Sería irrealista esperar que aquéllos cuyos intereses son servidos por el desarrollo dependiente cedieran sus privilegios sin un enfrentamiento. Sin embargo, lo que la zona de desarrollo puede ofrecer es una forma de escapar del horrible dilema entre la dependencia sin desarrollo y el desarrollo capitalista sin justicia social. En la medida en que las maquiladoras de Tijuana, Juárez o Matamoros, estén creando las condiciones para zonas de desarrollo genuinas, éstas representarán una estrategia viable de desarrollo; sin embargo, como he señalado, actualmente quizá estén haciendo más por Estados Unidos que por México. 


\section{B I B L I O G R A F I A}

ADIKIBI, O. 1983. "The transfer of technology to Nigeria: the case of tyre production". En: C. Kirkpatrick,C. y F. Nixson, eds. The industrialization of less developed countries. Manchester, Manchester, University Press.

BAIRD, P. y E. McCaughnan. 1979 Beyond the border. New York, NACLA.

CARRILLO, J. 1985. Conflictos laborales en la industria maquiladora. Tijuana, CEFNOMEX.

CARRILLO, J.y Mónica Jasis. 1983. "La salud y la mujer obrera en las plantas maquiladoras. El caso de Tijuana". Tijuana, CEFNOMEX.

CLEMENT, N. y S. Jenner. 1987. "Location decisions regarding maquiladora, in-bond plants operating in Baja California, Mexico".

DORAN, C. et al. eds. 1983 North/South relations: studies of dependency reversal. New York, Pracger.

EVANS, P. 1979. Dependent development: the alliance of multinational, state, and local capital in Brazil. Princeton, Princeton University Press.

FRIEDMAN, J., \& C. Weaver, 1979. Territory and function. Londres, Edward Arnold.

FROBEL, F., J. Heinrichs \& O. Kreye. 1980. The new international division of labour. Cambridge, C.U.P.

GOBSON, L.J. y A. Corona Rentería, eds. 1985. The U.S. and Mexico: borderland development and national economies. Boulder, Westview Press.

GRITSCH, N. 1986. “Mexico's maquiladoras examined: are in-bonD production plants the wave of the future?"Peperdine Law Review No. 13.

LAZES, P. 1986. Trico Productos Corporation Studies. New York, Coronell University Programs for Employment and Workplace Systems.

LIM, Linda. 1985. Women workers in multinational enterprises in developing countries. Geneva. ILO.

MAXFIELD, S. \& R. Anzaldua M. (eds). 1987. Government and private sector in contempoary Mexico. San Diego, Center for US-Mexico Studies. UCSD. 
RUIZ, V. y S. Tiano, (por aparecer). Women on the United States Mexico border: Responses to change. London, Allen and Unwin.

SKLAIR, L. 1985. "Shenzhen: a chinese 'development zone' in global perspective".En: Development change Vol. 16.

SKLAIR, L. (por aparecer A) "The costs of foreing investment: the case of the egyptian free zones". En: Middle Eastern Studies".

SKLAIR, L. (por aparecer B) "Foreing investment, irish development and the international division of labour". Progress in planning.

SKLAR, R. 1976."Postimperialism: a class analysis of multinational corporate expansion". Comparative politics No. 9.

UNIDO. 1971. Industrial free zones as incentives to promote export-oriented industries. Viena, United Nations Industrial Development Organization.

UNIDO 1980. Export processing zones in developing countries. Viena. UNIDO. 